\title{
UXUG-AP - Diretrizes de Experiência do Usuário e Usabilidade para Projeto Ágil
}

\author{
Aline de Oliveira Sousa ${ }^{\dagger}$ \\ Departamento de Informática \\ Universidade Federal do Paraná \\ Curitiba Paraná Brasil \\ ninimec@gmail.com
}

\author{
Natasha Malveira Costa Valentim \\ Departamento de Informática \\ Universidade Federal do Paraná \\ Curitiba Paraná Brasil \\ natasha@inf.ufpr.br
}

\section{RESUMO}

Entregar um software de valor para os usuários é um desafio constante para os times que usam metodologias ágeis como um framework em seu processo de desenvolvimento de software. Muitos pesquisadores e profissionais tentam associar as metodologias ágeis com a usabilidade e a Experiência do Usuário (UX), a fim de criar um modelo que promova este tipo de entrega. Dessa forma, os estudos identificados na literatura apresentam tecnologias complexas que demandam muito conhecimento, custo, tempo e recursos. Com o objetivo de contribuir com esta linha de pesquisa, decidiu-se por apresentar uma integração simples, onde tempo, custo e recursos não se tornam obstáculos para a aplicação da técnica em vários formatos de times e metodologias ágeis. O principal objetivo deste Artigo é apresentar uma técnica de prototipação chamada "User Experience and Usability Guidelines for Agile Project" (UXUG-AP) que auxilia no projeto da usabilidade e UX, a qual poderia ser usada por times ágeis, independentemente da quantidade de recursos disponíveis, tamanhos de projetos e metodologias adotada.

\section{PALAVRAS CHAVE}

Projeto ágil, Usabilidade, Experiência do Usuário

\section{INTRODUÇÃO}

Atualmente, as pessoas estão acostumadas a uma tecnologia onipresente [3], ou seja, a tecnologia está inserida de várias formas no cotidiano das pessoas. Devido à pluralidade de softwares disponíveis, selecionar a melhor aplicação depende da qualidade.

A garantia de qualidade de um software é definida como uma abordagem sistemática para avaliar a qualidade do software, seus padrões, processos e procedimentos [7]. Dentre os critérios de qualidade estabelecidos nas ISO/IEC 25010 [8] e ISO/IEC 9241210 [9], são a usabilidade e a experiência do usuário (UX).

Koivuniemi [3] aponta que o projeto da UX começa com uma boa usabilidade, fazendo o produto fácil e eficiente para o uso.

Permission to reproduce or distribute, in whole or in part, material extracted from this work, verbatim, adapted or remixed, as well as the creation or production from the content of such work, is granted without fee for non-commercial use, provided that the original work is properly credited. IHC 2019 - WORKSHOP DE TESES E DISSERTAÇÕES, Outubro 2125, 2019, Vitória, Brasil. In Anais Estendidos do XVIII Simpósio Brasileiro sobre Fatores Humanos em Sistemas Computacionais. Porto Alegre: SBC. (C) 2019 by the author(s), in accordance with the terms of the Creative Commons Attribution-NonCommercial 4.0 International Public License (CC BY-NC 4.0).
Contudo, tanto a usabilidade, quanto a UX vão muito além, presando pela satisfação do usuário, atendendo suas necessidades de forma eficaz. Dessa forma, considerar a usabilidade e a UX no software tem se apresentado como um dos desafios da área de Interação Humano-Computador (IHC).

Diante dessa necessidade, identificou-se que muitos profissionais buscaram melhorar o processo ágil integrando conceitos de usabilidade e/ou UX. Contudo, estas integrações apresentam-se de forma complexa, além de muitas vezes dirigir-se a metodologias ágeis específicas. Devido a esses fatores, o principal objetivo desta pesquisa é desenvolver uma técnica, ou seja, um conjunto de passos que visam alcançar um determinado objetivo [34], que apoie a construção de protótipos visando a usabilidade e UX dentro de um processo de desenvolvimento ágil.

Para tanto, este artigo apresenta a versão inicial da técnica User Experience and Usability Guidelines for Agile Project (UXUGAP) que possui um conjunto de diretrizes com a finalidade de apoiar o projeto da usabilidade e UX durante a prototipação.

Dessa forma, os trabalhos relacionados e o problema são apresentados na seção 2 . Na seção 3 apresenta-se a metodologia utilizada, enquanto a seção 4 apresenta as atividades já executadas. A seção 5 apresenta a contribuição esperada com a proposta e a seção 6 comenta sobre os próximos passos e considerações finais.

\section{TRABALHOS RELACIONADOS E APRESENTAÇÃO DO PROBLEMA}

Inúmeros profissionais vêm apresentando novas formas de tornar o processo de desenvolvimento de software mais simples, ao mesmo tempo que visam entregar maior qualidade. Muitas pesquisas têm sido desenvolvidas visando encontrar formas de unificar metodologias ágeis aos conceitos de usabilidade e UX [12] [24] [11] [25] [23] com a finalidade de melhorar o valor do produto, mantendo a agilidade da entrega.

Embora muitos pesquisadores estejam trabalhando na integração desses conceitos, poucos são aqueles que focam na integração tanto da usabilidade, quanto da UX, dentro do desenvolvimento ágil [12] [28]. Parte deles apresentam formas de integrar apenas a usabilidade ao processo de desenvolvimento ágil [29] [30] [27], outras integram apenas a UX [12] [26] [17].

Além disso, boa parte das pesquisas apresentam frameworks, metodologias e conjuntos de técnicas [1] [15] [18] [23] [25] [31], sendo esses tratamentos mais complexos, podendo gerar dependência de treinamento, exigir alguma habilidade específica dos profissionais, ou demandar mais tempo na execução. Em 
adição, algumas tecnologias são construídas com base em metodologias específicas, como o XP [15] [18]. Não obstante, por se tratar de uma demanda recente, ainda é necessário validar empiricamente estas integrações [17].

Com base nisso, surgiu a seguinte questão de pesquisa: Como projetar usabilidade e UX, dentro de um contexto ágil, de forma fácil, flexível, sem aumento de tempo, custo e recursos?

\section{METODOLOGIA DA PESQUISA}

A fim de desenvolver e avaliar uma solução para o problema proposto, a pesquisa foi dividida em duas etapas: concepção e avaliação, conforme pode ser visto na Figura 1. Durante a concepção realizou-se o Mapeamento Sistemático da Literatura, o qual concedeu a base teórica que fundamentou a construção da técnica proposta, além da análise dos estudos resultantes.

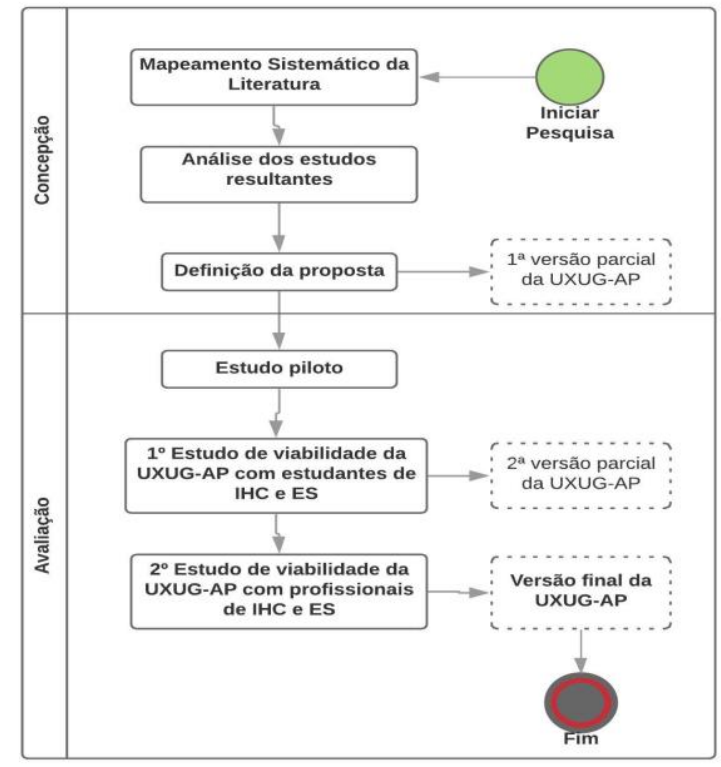

Figura 1 - Metodologia da Pesquisa

Já a etapa de avaliação, agora em andamento, iniciou-se pelo estudo piloto, que forneceu indícios que os materiais criados para a técnica são válidos. Este estudo será seguido por dois estudos de viabilidade, sendo um deles tendo como participantes estudantes da graduação e o outro, profissionais da área. Ambos deverão fornecer insumos visando a melhoria e evolução da técnica.

\section{ATIVIDADES REALIZADAS}

\subsection{Mapeamento Sistemático da Literatura}

Foi realizado um Mapeamento Sistemático da Literatura (MSL), seguindo os procedimentos descritos por Kitchenham e Charters [4]. O MSL teve como objetivo, segundo a abordagem Goal Question Metric (GQM), analisar publicações científicas, com o propósito de caracterizar as tecnologias de IHC e/ou Engenharia de Software (ES) que apoiam o projeto de usabilidade e UX, do ponto de vista de pesquisadores de IHC e ES, sob o contexto das fontes primárias em SCOPUS, ACM, IEEE Xplore e Science Direct.
Neste trabalho, o termo "tecnologia" é utilizado como generalização para procedimentos, ferramentas, técnicas, metodologias e outras propostas realizadas na área de IHC e Engenharia de Software [32].

O MSL buscou responder a seguinte questão: "Quais tecnologias têm sido utilizadas nas fases iniciais do processo de desenvolvimento ágil de um software, visando projetar a usabilidade e/ou UX? ". Afim de auxiliar na resposta da questão da pesquisa foram definidas as seguintes sub questões (SQ):

SQ1: Qual o estágio do processo de desenvolvimento ágil?

SQ2: Qual a área da pesquisa?

SQ3: Quais os critérios de qualidade da tecnologia?

SQ4: Quais os artefatos em que a tecnologia é aplicada?

SQ5: Qual o tipo de contribuição?

SQ6: Necessita de suporte ferramental?

SQ7: Foram realizados estudos empíricos?

SQ7.1: Quais os tipos de estudos empíricos?

SQ7.2: Qual o ambiente de avaliação do estudo realizado?

Além disso, utilizou-se o PICOC (Population, Intervention, Comparison, Outcome e Context) para identificar as palavras-chave e formular a string de busca, a qual ficou definida como:

("agile development" OR "agile project" OR "agile lifecycle" OR "agile method") AND ("technique" OR "tool" OR "framework" $\mathbf{O R}$ "method" $\mathbf{O R}$ "model" $\mathbf{O R}$ "process" $\mathbf{O R}$ "guideline" OR "practice" OR "approach" OR "principle" OR "heuristic") AND ("usability design" OR "user experience design" OR "UX design")

Foram definidos critérios para filtrar os trabalhos retornados pela busca. Sendo os critérios de inclusão (CI):

CI1: Estudos que descrevam tecnologias que projetam a usabilidade e experiência do usuário em fases iniciais dos processos de desenvolvimento ágil;

CI2: Estudos empíricos de tecnologias que projetam a usabilidade e experiência do usuário em fases iniciais dos processos de desenvolvimento ágil;

E os critérios de exclusão (CE):

CE1: Livros, chamadas de conferência, relatórios técnicos;

CE2: Os artigos que não atendam aos critérios de inclusão;

CE3: Os artigos em que a língua é diferente do inglês e do português;

CE4: O artigo não está disponível para leitura (disponíveis através de pagamento, ou não fornecidos pelas bibliotecas);

CE5: O artigo é duplicado.

Após realizar a busca nas bibliotecas definidas, a seleção dos artigos foi realizada em duas etapas. $\mathrm{O} 1^{\circ}$ Filtro, consistiu na leitura do título e abstract, filtrando os artigos de acordo com os critérios de inclusão e exclusão. No $2^{\circ}$ Filtro foi realizada a leitura completa dos artigos resultantes, novamente filtrando-os de acordo com os critérios definidos. Ambas as etapas contaram com duas pesquisadoras, onde a primeira fazia o primeiro crivo e, a pesquisadora mais experiente revisava, fazendo as devidas alterações quando necessário. No total, 232 artigos foram retornados pelas bibliotecas digitais. Ao final do $1^{\circ}$ filtro, 124 artigos foram selecionados e no $2^{\circ}$ filtro chegou-se ao total de 18 artigos que passaram pela extração dos dados. 
UXUG-AP - User Experience and Usability Guidelines for Agile Project

\subsection{Análise dos Estudos Resultantes}

Foi realizada uma análise dentre os artigos resultantes do MSL, a fim de verificar quais poderiam contribuir de alguma forma com a construção da técnica. Inicialmente decidiu-se por utilizar a técnica de prototipação como base, por ter sido a técnica mais mencionada (11 menções), além de apresentar características convenientes para a proposta, como facilidade, flexibilidade e baixo custo. Depois, foram estabelecidos dois critérios para seleção: o artigo deveria mencionar a técnica de prototipação e ao mencioná-la, deveria conter informações relevantes sobre sua construção e/ou utilização no dado projeto. Dos 18 artigos resultantes, somente 2 foram selecionados para auxiliar na construção da técnica, sendo eles [18] e [15], apresentados resumidamente a seguir.

Hussain et al. [15] apresentaram um processo baseado em Interação Humano-Computador (IHC) aplicado em um projeto que utilizava a metodologia XP. O processo consistia em associar 5 instrumentos de IHC à metodologia XP. A conclusão do estudo destacou que, o sucesso da união entre o ágil e o design centrado no usuário depende principalmente do usuário e sua participação no projeto.

Memmel et al. [18] apresentaram um estudo onde foi aplicado o ciclo de vida XP estendido pela engenharia de usabilidade. O ciclo de vida priorizava as questões interativas e funcionais, tratando-as antecipadamente. A abordagem também privilegia o uso de protótipos expressivos, com a justificativa de diminuir a necessidade de usar documentos abstratos e extensivos.

\subsection{UXUG-AP}

A técnica chamada User Experience and Usability Guideline for Agile Project (UXUG-AP) [22] visa apoiar o projeto da usabilidade e UX durante a construção dos protótipos em um processo de desenvolvimento ágil. A UXUG-AP pretende promover a integração das disciplinas de usabilidade, experiência do usuário e metodologias ágeis, sob o formato de diretrizes. Com a finalidade de direcionar o profissional a desenvolver uma ideia sistemática da aplicação, as categorias passam por tópicos como requisitos, tipos de usuários, indo até detalhes como separação de informações e prevenção de erros, conforme pode ser visto na Tabela 1. Cada categoria se divide em subcategorias, de forma a permitir uma leitura rápida e simples do tópico de interesse. Além disso, essa divisão em categorias e subcategorias partiu da ideia de que o profissional teria inicialmente, a liberdade em escolher quais características ele gostaria de usar e aplicar em seu projeto.

Desta forma, a técnica proporciona a flexibilidade e autonomia necessárias as várias metodologias ágeis existentes. Além disso, a técnica apresenta exemplos que ilustram a diretriz, permitindo ao profissional um melhor entendimento, facilitando sua utilização.

Além das obtidas no MSL, houve a necessidade de buscar outras referências para compor a técnica. Com isso, incluiu-se a ISO/IEC 25010 [8], além de outras referências como [10], [6], [19], [21], [5], e [2]. Finalmente, definiu-se a UXUG-AP [22], apresentando-a numa estrutura linkada, de forma que o profissional possa navegar livremente, conforme a necessidade.
WTD-IHC 2019, Outubro, 2019, Vitória, Espírito Santo - Brasil

Tabela 1 - Categorias e Subcategorias da UXUG-AP

\begin{tabular}{|c|c|}
\hline Categorias & Subcategorias \\
\hline \multirow{3}{*}{ Requisitos } & Troca de Informações - Time/Cliente \\
\hline & Entrevistas e Workshops \\
\hline & Requisitos Chave \\
\hline \multirow{3}{*}{$\begin{array}{lr}\text { Entendimento } & \text { das } \\
\text { necessidades } & \text { do } \\
\text { usuário } & \end{array}$} & Iniciantes e Especialistas \\
\hline & Crianças, Jovens, Adultos e Idosos \\
\hline & Leigos, Acadêmicos e Profissionais \\
\hline \multirow{2}{*}{ Acessibilidade } & Deficientes Visuais I \\
\hline & Deficientes Visuais II \\
\hline Facilidade no uso & Localização \\
\hline \multirow{4}{*}{$\begin{array}{l}\text { Feedback } \\
\text { informativo }\end{array}$} & Mensagens de Confirmação e Alerta \\
\hline & Mensagens de Erro \\
\hline & Componentes de Carregamento \\
\hline & Títulos e Links \\
\hline \multirow{3}{*}{ Prevenção de erros } & Obrigatoriedade dos Campos \\
\hline & Apresentação Autoexplicativa \\
\hline & Limitando Campos \\
\hline \multirow{2}{*}{$\begin{array}{l}\text { Agrupamento de } \\
\text { informação }\end{array}$} & Independência de Informação \\
\hline & Modularização de Informação \\
\hline \multirow{2}{*}{ Sequência de Ações } & Organização das Ações Sequenciais \\
\hline & Comportamento das ações Sequenciais \\
\hline $\begin{array}{ll}\text { Sentimento } & \text { de } \\
\text { Pertencimento } & \\
\end{array}$ & Conexão Emocional \\
\hline \multirow{2}{*}{ Grau de Importância } & Disposição das Informações \\
\hline & Termos Relevantes \\
\hline \multirow{2}{*}{ Privacidade } & Controle de Informação \\
\hline & Senhas \\
\hline
\end{tabular}

\subsection{Estudo Piloto}

O estudo piloto foi realizado com o propósito de validar os artefatos utilizados durante o experimento, bem como a viabilidade da técnica. Para a realização do estudo, foi solicitado aos participantes que construíssem protótipos do tipo Web Page, com base em uma estória do usuário dada.

Após a construção dos protótipos foi entregue aos participantes um questionário pós-uso, baseado no modelo Technology Acceptante Model (TAM) [33], a fim de avaliar a UXUG-AP. Os participantes avaliaram a técnica com base em três fatores: Facilidade no uso, Utilidade e Futuro uso da UXUG-AP. Através da análise realizada, verificou-se que, os participantes avaliaram a UXUG-AP como parcialmente fácil de usar, atendendo ao objetivo proposto, porém exigindo um certo esforço mental, o qual associaram a falta de conhecimento no assunto.

Sobre a utilidade, ambos também consideraram que a UXUGAP aumentou o desempenho, produtividade e eficácia durante a prototipação. Um dos participantes ainda destacou que a técnica o ajudou nas questões que envolviam UX e acessibilidade, visto que o mesmo não possuía conhecimento no assunto. Já a respeito da intenção de uso futuro, um participante destacou que, com a evolução da técnica, a mesma seria muito bem recomendada. 


\section{CONTRIBUIÇÃO ESPERADA}

Com esta pesquisa, espera-se desenvolver uma técnica que dê o suporte necessário aos profissionais que atuam em times ágeis, de forma que os processos de construção dos artefatos de prototipação contemplem também os requisitos de usabilidade e experiência do usuário. Dessa forma, a principal contribuição que se espera alcançar é apresentar a indústria uma forma alternativa de prototipação que possibilite diminuir o retrabalho e custos dos projetos, prevenindo-os de possíveis problemas ou falhas de usabilidade e experiência do usuário que, normalmente, são encontrados somente ao final do processo, ou após a entrega.

\section{PRÓXIMOS PASSOS}

Recentemente aplicou-se o primeiro estudo de viabilidade, agora em estado de análise. Com base nos resultados do primeiro estudo, deseja-se evoluir a técnica, para então validá-la no segundo estudo de viabilidade que será realizado com profissionais que trabalhem com metodologias ágeis. Após a realização dos estudos de viabilidades e suas respectivas análises, espera-se alcançar uma técnica consistente e que seja capaz de auxiliar as equipes ágeis a construírem softwares com usabilidade e UX, de forma fácil e sem custos adicionais.

\section{AGRADECIMENTOS}

As autoras agradecem o apoio do Departamento de Informática da UFPR, bem como a participação dos alunos da pós-graduação no estudo piloto e também dos alunos da graduação para a execução do primeiro estudo de viabilidade.

\section{REFERÊNCIAS}

[1] A.Y. Wale-Kolade, 2015. Integrating usability work into a large interorganizational agile development project: Tactics developed by usability designers. The journal of Systems and Software, pp. 54-66, 2015.

[2] A. Harley. UX Guidelines for Recommended Content. Disponível em $<$ https://www.nngroup.com/articles/recommendation-guidelines/>. Acesso em: 07 de janeiro de 2019.

[3] A. Koivuniemi, 2013. Developing usability method for agile software development - Case study on RISE for Traffica. Universidade de Tempere. 2013.

[4] B. Kitchenham, S. Charters, 2007. Guidelines for performing Systematic Literature Reviews in Software Engineering. EBSE 2007-001.Keele University and Durham University. Joint Report. 2007.

[5] B. Tognazzini, 2019. First Principles of Interaction Design (Revised \&Expanded). Disponível em $<$ https://www.nngroup.com/articles/firstprinciples-interaction-design/> . Acesso em: 07 de janeiro de 2019.

[6] B. Scheneiderman, C. Plaisant, M. Cohen, S. Jacobs, N. Elmgvist. Designing the User Interface: Strategies for Effective Human-Computer Interaction. $6^{a}$ Edição, Pearson (May 2016). Disponível em <http://www.cs.umd.edu/hcil/DTUI6/> Acesso em: 07 de janeiro de 2019.

[7] C. SenthilMulrugan, S. Prakasam, 2013. International Journal of Conputer Applications, vol.78, $\mathrm{n}^{\circ}$ 8. Setembro, 2013.

[8] ISO/IEC, 2011. ISO/IEC 25010: Systems and software engineering - SquaRE Software Product Quality Requirements and Evaluation: System and Software Quality Models. 2011.

[9] ISO/IEC, 2010. ISO/IEC 9241-210: International Standardization Organization (ISO).Ergonomics of human system interaction- Part 210: Human-centered design for interactive systems. 2010.

[10] L. Carvajal, A.N. Moreno, M. I. Sanchez-Segura, A. Seffah 2013. Usability through Software Design. IEEE Transactions Software Engineering, vol. 39, n11, Novembro, 2013.

[11] M. Maguire, 2013. Using human factors standards to support user experience and agile design. Stephanidis, C. and Antona, M. (eds.). $7^{\mathrm{a}}$ International UAHCI Conference, HCI International, Las Vegas, USA, 21-26 July 2013, pp. 185-194.
[12] M. Isomursu, A. Sirotkin, P. Voltti, M. Halonen, 2012. User Experience Design Goes Agile in Lean Transformation - A Case Study, in 2012 Agile Conference, pp. 1-10, Dallas, Texas. 2012

[13] O. Almughram, S. Alyahya, 2017. Coordination Support for Integrating User Centered Design in Distributed Agile Projects. SERA 2017. IEEE. pp. 229-238. Londres. Reino Unido. 2017.

[14] P. Hodgetts, 2005. Experiencies Integrating Sophisticated User Experience Design Practices into Agile Project. Agile Development Cnference. Fullerton, Califórnia, 2005.

[15] R. Newhook, D. Jaramillo, J.G. Temple, K.J. Duke, 2015. Evolutions of the mobile enterprise app: A design perspective. ${ }^{\text {a }}$ International Conference on Applied HumanFactors and Ergonomics and Affiliated Conferences. Procedia Manufacturing 3, pp. 2026-203. 2015.

[16] S.H. Lee, L.Y. Ko, S. Kung, D.H. Lee, 2010. A Usability-patern-based Requirements-analysis Method to Bridge the Gap between User Tasks and Application Features. 34 $4^{\text {th }}$ Annual Computer Software and Applications Conference. pp. 317-326. 2010.

[17] T.S.D. Silva, M.S. Silveira, F.F. Silveira, 2007. The evolution of Agile UXD. Information and Software Technology, vol. 102, pp. 1-5. Outubro, 2018.

[18] T. Memmel, H. Reiterer, A. Holzinger, 2007. Agile Methods and Visual Specification in Software Development: A Chance to Ensure Universal Access. 2007 HCI Conference, pp.453-462. 2007.

[19] Usability Guidelines, 2016. U.S. Departament of Health \& Human Services. Disponível em < https://webstandards.hhs.gov/guidelines/>. Acessado em $20 \mathrm{de}$ Junho de 2018.

[20] V. Paelke, k. Nebe, 2008. Integrating Agile Methods for mixed RealityDesign Space Exploration. $7^{\mathrm{a}}$ ACMConference on Designing Interactive Systems. pp.240-249. Cape Tow, África do Sul. 2008.

[21] Windows User Experience Interaction Guideline, 2019. Microsoft Corporation. Disponível em $<\quad$ https://docs.microsoft.com/enus/windows/desktop/uxguide/guidelines>. Acesso em: 07 de Janeiro de 2019.

[22] Técnica UXUG-AP - User Experience and Usability Guidelines for Agile Project Disponível em

https://www.dropbox.com/home/Pesquisa_Mestrado_AlineSousa/5_Experiment os/2_Documentos_UXUG-AP?preview=UXUG-AP.pdf > Acesso em: 02 de Julho de 2019.

[23] Z. Hussain, M. Lechner, H. Milchrahm, S. Shahzad, W. Slany, . Umgeher, T. Vlk, C. Köfel, M. Tscheligi, P. Wolkerstorfer, 2012. Practical Usability in XP Software Development Process. The Fifth International Conference on Advancesin Computer-Human Interactions. pp. 208-217. Valencia, Spain, 2012

[24] D. Teka, Y. Dittrichy, M. Kifle, 2017. Contextualized User Centered DesignWith Agile Methods in Ethiopia.IEEEAFRICON 2017. pp. 911-916. 2017.

[25] T. Pfeiffer, J. Hellmers, E. M. Shön, J. Thomaschewski, 2016. EmpoweringUser Interface for Industrie 4.0. Proceeddinsg of IEEE, Vol 4, N 5, 2016.

[26] N. Karamanis, M. Pignatelli, D. Silva-Carvalho, F. Rowland, J.A. Cham, I. Dunhan, 2018. Designing nad Intuitive Web Application for Drug DiscoveryScientists. Drug Discovery Today, vol 3, pp. 1169-1174. Junho, 2018.

[27] O. Sohaib, k. Khan, 2010. Integrating Usability Engineering and Agile Software Development: A Literature Review. Computer Design and Applications (ICCDA), 2010 International Conference on, Qinhuangdao, 2010, pp. V2-32V2-38.

[28] J. Highsmith. Agile SoftwareDevelopment Ecosystems. Adisson-Wesley, 2002.

[29] J.C. Lee, D.S. McCrikard. Towards Extreme (ly) Usable Software: Exploring Tensions Between Usability and Agile SoftwareDevelopment. 2007 Agile Conference, pp.59-71. Washington, DC, USA, 2007.

[30] M.Singh. U-SCRUM: Na Agile Methodology for Promoting Usability. 2008 Agile Conference, pp.555-560.Toronto, Ontário Canadá, 2008.

[31] E.M Schön, J. Thomaschewski, M.J. Escalona. Agile Requirements Engineering: A systematic literature review. Computer Standards \& Interfaces, vol. 49, pp.7991, Janeiro, 2017.

[32] G. Santos, A. Rocha, T. Conte, M. Barcellos, R. Prikladnicki. Strategic Alignment between Academy and Industry: A Virtuous Cycle to Promote Innovation in Technology. In Brazilian Symposium on Software Engineering, Natal, v. 1, pp. 196 - 200, 2012.[33] F.D. Davis, R.P. Bagozzi, P.R. Warshaw. User acceptance of computer technology: A comparison of two theoretical models. Management Science, 35 (8): 982-1003, 1989.

[34] José., Ortega y Gasset. Meditação sobre a técnica. [S.1.]: Instituto Liberal. 1991. 